\title{
Dynamic behavior and damping capacity of auxetic foam pads
}

\author{
F. Scarpa, J. A. Giacomin, A. Bezazi and W. A. Bullough \\ Department of Aerospace Engineering, University of Bristol, BS8 1TR Bristol, UK. Email: \\ f.scarpa@bristol.ac.uk \\ Perception Enhancement Group, Brunel University, UB8 3PH Uxbridge, UK. \\ Department of Mechanical Engineering, University of Sheffield, S1 3JD Sheffield, UK
}

\begin{abstract}
A novel set of auxetic (negative Poisson's ratio) open cell polyurethane foam has been developed and tested under dynamic loading conditions to assess the viscoelastic response under white noise random excitation and compressive cycling. Foam pads normalized to standard ISO 13753 have been tested at room temperature and frequency bandwidth 10-500 $\mathrm{Hz}$ to assess transmissibility characteristics for possible antivibration glove applications. The results show that the ISO 13753 normalized transmissibility for these foams falls below 0.6 above $100 \mathrm{~Hz}$, with lower peak maximum stresses under indentation compared to conventional open cell solids. These results suggest possible use of the auxetic foam for pads or linens against « white fingers « vibration applications. Further tests have been conducted on cyclic compressive loading up to $3 \mathrm{~Hz}$ and loading ratios of $0.95 \mathrm{for}$ loading histories up to 100000 cycles. The damping capacity of the auxetic foams showed and increase by a factor 10 compared to the conventional foams used to manufacture the negative Poisson's ratio ones, and stiffness degradation stabilized after few tens on cycles.
\end{abstract}

Keywords: Auxetic, open cell foams, vibration, transmissibility, cyclic loading, damping capacity

\section{Introduction}

Since their introduction in 1987 [1], there has been a recent surge of interest in auxetic (negative Poisson's ratio) materials for a wide range of engineering applications, from damage tolerant laminates [2], microwave absorbers [3] and medical prosthesis [4]. The large number of applications is related to the peculiar deformation mechanisms of auxetic configurations. In fact, and auxetic (negative Poisson's ratio) materials expand in all directions when pulled in only one, leading to an unusual volume change of the solid itself. The negative Poisson's ratio behaviour does not contradict the classical theory of elasticity. A homogeneous, isotropic, thermodynamically correct 3D solid has a potential Poisson's ratio range between -1.0 and 0.5 , while the magnitude of the Poisson's ratio can be even larger in the case of anisotropic solids. The dynamic behaviour of auxetic materials has been investigated by several authors $[\mathbf{5}, \mathbf{6}, \mathbf{7}, \mathbf{8}]$. The possible use of auxetic materials for viscoelastic damping applications has been examined in [9], where biphasic auxetic composite showed loss tangent exceeding lower Voigt limit, and close to Hashin upper bound. In recent papers [10,11] increases in loss factor and storage moduli have been shown for auxetic PU foams manufactured using a modified route from the classical production layout.

In the field of vibration absorber pads, testing of antivibration glove cushioning materials has been the subject of several research studies [12,13] which have lead to the establishment ISO standard 13753:1999 [14], which defines a method for measuring the mechanical transmissibility of glove cushioning materials. The method determines the impedance of the material when loaded by a mass providing a compression force equivalent to that found when the material is gripped by the hand. This is done by measuring the transfer function of the mass-loaded material at all the required frequencies. The vibration transmission when loaded by the hand is computed using standard values of hand-arm impedance and the measured values of the material impedance. The impedances used in this International Standard are for the palm of the hand when gripping a circular handle. ISO 13753:1999 provides a useful, and relatively simple, method for evaluating the isolation properties of resilient materials for antivibration gloves or other products which attempt to reduce the transmission of vibration to the human hand-arm system.

The cyclic stress-strain behaviour of polymer foams and elastomers has attracted recent attention, particularly when damping ability is considered, since both types of material have good vibration absorption qualities. When samples are loaded under strain control and then unloaded, subsequent extension to the same strain requires a lower force. Further cycling results in continued softening at a progressively slower rate and a steady state may be reached. This softening phenomenon is an important indication of the amount of energy that the material can continue to absorb. A polyurethane foam open cell subjected to compressive cyclic until 100 cycles has been investigated by Rhekopf et al.[15]. Shen et al. [16] proposed a model based on the experimental results in [15] which could be applied to express the cyclic stress-strain relationship for conventional polyurethane foam at any given cycle. Polymeric foams are viscoelastic materials, and the rate of loading, or frequency of the load cycles, become important factors to consider. In sandwich structures with polymeric foam core, it is expected that the viscoelastic behavior of the foam play an important role in absorbing and dissipating energy especially during dynamic loading [17]. Such dynamic loading could be in terms of cyclic or high strain rate loading during impact.

Both vibration transmissibility and damping capacity under repeated cyclic loading are important issues on selecting foam materials for applications where both vibroacoustics and structural integrity targets have to be met. While for positive Poisson's ratio materials the analysis of viscoelastic core properties (in particular for sandwich pads and structures applications) has been considered in few studies $[\mathbf{1 8}, \mathbf{1 9}]$, there is even more scarcity of available results for auxetic open cell PU-PE foams. In this paper we presented a combined series of experimental results on negative Poisson's ratio open cell foams, both from a vibration and cyclic fatigue point of view. As it will be evident, these new types of foams offer multiple advantages in terms of transmissibility reduction above $100 \mathrm{~Hz}-150 \mathrm{~Hz}$ (where "white fingers" problems become serious issues in hand-held devices), indentation resistance, stability of stiffness under compressive cyclic loading, and damping capacity for energy absorption under repeated compressive forces.. 


\section{Manufacturing of the auxetic foams and Poisson's ratio measurement}

The specimens that were tested were produced from conventional grey open-cells polyurethane foam with 30-35 pores/inch and $0.0027 \mathrm{~g} / \mathrm{cm}^{3}$ density, supplied by the McMaster-Carr Co. of Chicago Illinois. The manufacturing process followed a procedure outlined in [20]. An aluminium mould consisting of cylinders backed by pistons was used to impose concurrent axial and radial compression on the parent foam samples. The compression ratio value ranged from 3 to 6 along the two manufacturing directions, followed by heating until the softening temperature was reached. The base foam was supplied in squared blocks of $600 \mathrm{~mm}$ side by $50 \mathrm{~mm}$ thickness. Cylindrical samples were obtained using a sharp-edge tube which was penetrated into the block of conventional foam. These samples were then inserted inside the lubricated tubes, using a conical inlet to avoid creases. The tubes containing the parent foam were then positioned in the mould frame and the piston pushed inside it. The locking of the piston in the desired position followed compression. The whole operation was then repeated for the other specimens. The mould was then put into an industrial oven, and two heating profiles [20] were used. The heating process was followed by room temperature cooling, which lasted two hours on average. After this operation, the extracted specimens were gently tensioned in order to relax the external surface. Following a procedure outlined in [20], the general designation of the samples was defined by an "XY" code, where " $\mathrm{X}$ " was the position of the specimen in the mould and " $\mathrm{Y}$ " was the progressive letter assigned to every specimen. Samples with higher uniformity of mechanical properties belonged to the specimens C and L (parameters shown in Table 1). For comparison, specimens with no imposed radial compression were also tested. These latter manufacturing parameters were labelled as "M" specimens. The specimens with no imposed radial load were produced as a control sample, to check against the possibility that measured changes in mechanical and transmissibility properties of the auxetic foams were due to the increase in foam density.

In a manner similar to the procedure outlined in [20, 21], Poisson's ratio measurements were based on image data acquired with a SONY DCR-TRV50E digital camera and processed with the MATLAB v6.1 software. The images were acquired during quasi-static tests performed using an MTS 858 servo-hydraulic machine. The Poisson ratio measures were carried out using specimens which were cut to $40 \mathrm{~mm}$ in length and glued to the end clamp of the machine with Loctite ${ }^{\circ}$ adhesive, then stretched until breaking or ungluing at $0.1 \mathrm{~mm} / \mathrm{s}\left(\dot{\mathrm{e}}=0.0025 \mathrm{~s}^{-1}\right)$. A calibration photo was taken for each test in order to convert the units of measurement from pixels to millimeters. A ruler was placed at a set distance from the camera iris, and the conversion factor was determined geometrically using the known distance of the ruler from the camera and the nominal focal length of the camera lens. After calibration, a set of photos was acquired to measure longitudinal and transverse strain during testing. This operation was performed using a MATLAB routine which calculated ten values of diameter and ten values of length along the specimen from every photograph, providing as output both the mean value and the standard deviation. To avoid end effects during the measurements, the extreme thirds of the dark-grey specimens were painted white in order to consider only the central third in the calculation of the lengths.

Figure 2 presents a comparison between the Poisson's ratio of the conventional foam and those of auxetic specimens 3C, 5C, 2L and $5 \mathrm{M}$. The Poisson's ratio values are all strain dependent, tending towards near-zero, or positive values, for large tensile strains. The foam of specimen $3 \mathrm{C}$ had its highest magnitude of Poisson's ratio for a tensile strain of approximately $90 \%$. For specimen 5C, the minimum Poisson's ratio values occurred at low tensile strains, with NPR average values of -0.22 . Beyond a tensile strain of $50 \%$, the Poisson's ratio of specimen 5C decreased with the applied strain, becoming positive at $100 \%$ elongation. Specimen $2 \mathrm{~L}$ presented a negative Poisson's ratio behaviour, with lower sensitivity to tensile strain, becoming near zero for tensile strains above $90 \%$. The iso-density foam 5M presented negative, albeit small, Poisson's ratio values. Theoretically, a zero radial compression should not be capable of generating a negative Poisson's ratio [4] in foam. In practice, during the manufacturing process the axial compression applied to the sample can be sufficient to push the foam specimen against the cylindrical walls of the mould, providing a radial compression of the specimen. It can be noted, however, that the values of the NPR are confined within the range -0.04 and -0.05 .

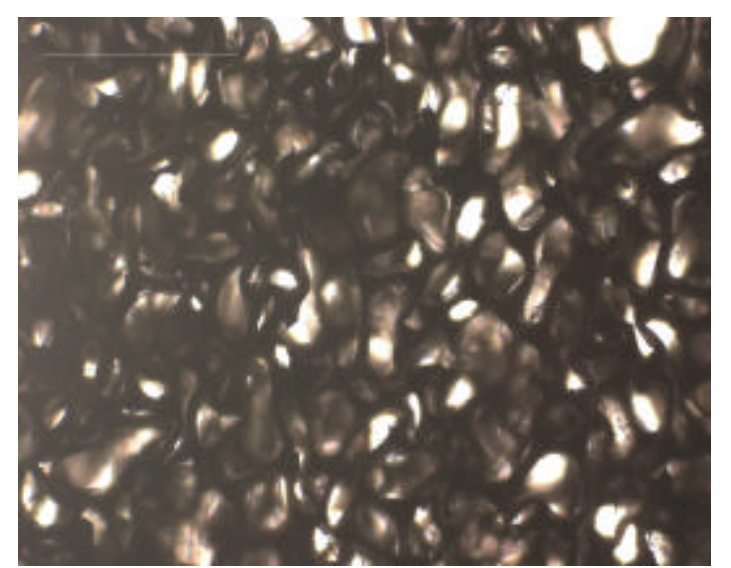

Figure 1. Microstructure of a typical auxetic foam

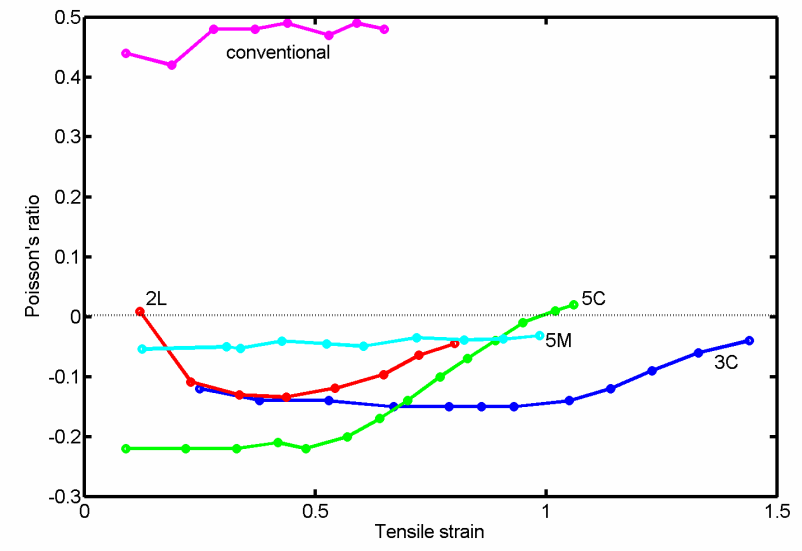

Figure 2. Poisson's ratios vs. tensile strains

\section{Dynamic tests}




\subsection{Transmissibility}

ISO 13753:1999 requires the measurement of the transfer function of the resilient material across the frequency range from 10 to $500 \mathrm{~Hz}$ while loaded by a steel cylinder which has a radius of $45 \mathrm{~mm}$ and a total mass of $2.5 \mathrm{~kg}$. The standard prescribes a material sample which is flat and of constant thickness, and which occupies the $45 \mathrm{~mm}$ radius area between the vibrating surface and the loading mass. For each individual band or frequency (between $10 \mathrm{~Hz}$ and $500 \mathrm{~Hz}$ ), the material impedance is calculated by means of the expression

$$
Z m=\frac{j \omega m}{\frac{A_{1}}{A_{2}}-1}
$$

Where $A_{1}$ is the complex amplitude of the input excitation and $A_{2}$ is the complex amplitude of the output excitation of the loading mass. The vibration transmission of the resilient material when loaded by the human hand is then estimated using standard values of hand-arm impedance which are provided in the standard, and the measured values of the material impedance from expression (1), as

$T=\frac{Z m}{Z h+Z m}$

In order to perform the ISO 13753:1999 measurements, circular samples were manufactured as shown in Figure 3. The samples presented a minimum overall diameter $D$ of $95 \mathrm{~mm}$, with the inner diameter $d$ equal to the one obtained from the aluminium mould. The thickness of each sample was $10 \mathrm{~mm}$. Samples were placed between the $2.5 \mathrm{~kg}$ loading mass and the base board, which had overall dimensions of $200 \mathrm{~mm} \mathrm{X} 200 \mathrm{~mm} \mathrm{X} 12 \mathrm{~mm}$ and which had a total mass of $3.74 \mathrm{~kg}$. The baseboard was connected to a rigid frame by bungee cords and springs having a total stiffness of $0.36 \mathrm{Nmm}^{-1}$. The excitation to the baseboard was provided by a V406 (SN 20333-2) electrodynamic shaker and accompanying power amplifier. A steel stinger rod with a 1 $\mathrm{mm}$ cross section was fixed to the baseboard with a screw-bolt system. Care was taken to align the stinger to the baseboard so as to guarantee a purely vertical motion of the baseboard. Two PCB Model 33C604 accelerometers were fixed to the loading mass and to the top of the baseboard using wax. The accelerometers (serial numbers 14582 and 14590) had calibration factors of $96.937 \mathrm{~m} / \mathrm{s}^{2} \cdot$ Volt and $94.966 \mathrm{~m} / \mathrm{s}^{2}$ respectively, and were amplified by a PCB Piezotronics Model 483A (SN 1355) amplifier. Figure 2 presents a schematic diagram of the laboratory test facility that was used. A DSP Siglab [22] software based on MATLAB 6.5 was used to drive the shaker and to perform the Discrete Fourier Transform (DFT) of the signals from the accelerometers. The shaker was driven using a random white noise signal with energy in the frequency range from 1 to $500 \mathrm{~Hz}$, and r.m.s. voltage amplitude of $1.4 \mathrm{~V}$, and r.m.s. acceleration at the mass head of $30 \mathrm{~g}$. The transmissibility of the material samples was calculated over the frequency range from 1 to $500 \mathrm{~Hz}$ using 1024 spectral lines and a $50 \%$ window overlap, with overload block rejection. Hanning windows were used for all data acquisitions.

Figure 4 presents the ISO 13753 transmissibility of the foams. Within the frequency range from 10 to $31.5 \mathrm{~Hz}$ it can be observed that all the transmissibility values were found to be greater than 1 , meaning that all the foams increased the magnitude of the transmitted vibration. This result is not unusual for thin, resilient, materials [23] in the hand-arm application. Over the 10 to 31.5 $\mathrm{Hz}$ frequency range the specimen $\mathrm{C}$ auxetic foam produced the highest transmissibility values, while foam specimen $2 \mathrm{~L}$ provided the lowest. For frequencies greater than $31.5 \mathrm{~Hz}$, all the foam specimens provided attenuation against vibration. It is worthy of note that although the auxetic L specimen provided better vibration isolation performance below $31.5 \mathrm{~Hz}$, the attenuation was lower compared to the conventional foam at higher frequencies. At the higher frequencies, the lowest transmissibility values were achieved by the lower manufacturing volumetric ratio auxetic foams (specimens C). For Example, at $100 \mathrm{~Hz}$ the conventional foam provided a transmissibility value of 0.9 , while the auxetic $5 \mathrm{C}$ foam provided a value of 0.8 . From this trend, it might be presumed that decreases in foam volumetric ratio produce changes in the transmissibility curve of the auxetic foam which move the curve in the direction of that of the corresponding conventional foam. If further research were to confirm this hypothesis, it would be possible to identify an optimised manufacturing volumetric ratio starting from a target transmissibility specification.

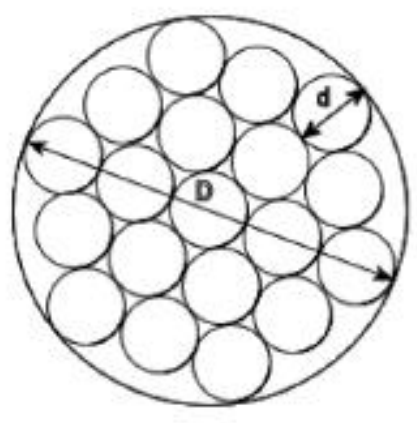

Figure 3. Layout of the specimens for ISO 13753

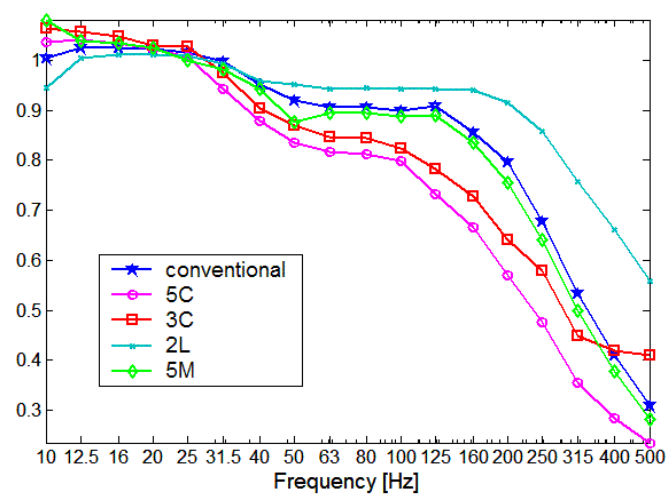

Figure 4. ISO 13753 transmissibility for different specimens 


\subsection{Compressive cyclic loading}

The fatigue tests have been performed in displacement control for all types of the foams with a sinusoidal waveform with $3 \mathrm{~Hz}$ of pulsation using the same MTS facility. The samples were preloaded at $70 \%$ of the maximal displacement obtained during the static tests, and then subjected to a range of different amplitudes (0.6, 1.2, 2.4 3.6, 4.8 and 6 mm), leading to different loading levels $r(0.725,0.75,0.80,0.85,0.90$ and 0.95$)$, where:

$$
r=\frac{u_{\max }}{u_{r}}
$$

Were $u_{\max }$ and $u_{r}$ are the maximum displacement and displacement at densification (80\% of the initial length). All tests were performed at room temperature $\left(18^{\circ} \mathrm{C}\right)$, with no specific humidity control.

A comparison of stiffness degradation (rigidity loss) between conventional, iso density and auxetic foams for different loading levels versus the number of cycles has been performed. It must be pointed out that, by iso density foam we consider the type 5M (i.e., similar volumetric compression ratio and density of the auxetic 5C, but with zero Poisson's ratio). The auxetic base foam we consider in these fatigue tests is the 5C type. For high loading levels $(r=0.95$ Fig. 5), the conventional foam shows enhanced resistance to fatigue, followed by the iso-density foam and finally by the auxetic one. The decrease on rigidity loss of the auxetic foam compared to the iso-density one amounts to $25 \%$ in this case. For lower loading ratio levels ( $\mathrm{r}=0.85$, Figure 6), the conventional and the iso density foams show very similar behaviour of stiffness ratio versus number of cycles, exhibiting an improved fatigue resistance compared to the auxetic one. The decrease of rigidity loss for the auxetic foam is $15 \%$ in this case. These results suggest that the auxetic foams are in general more sensible to compressive fatigue than the conventional and the iso density ones, this behaviour can be further evidenced by the loss of the energy.

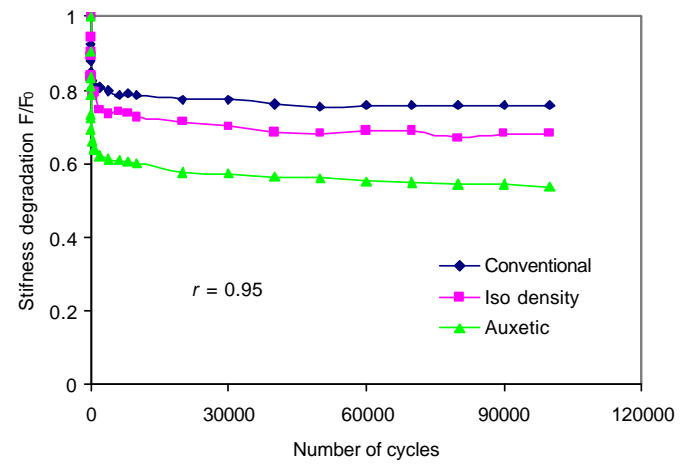

Figure 5. Stiffness degradation for $\boldsymbol{r}=\mathbf{0 . 9 5}$

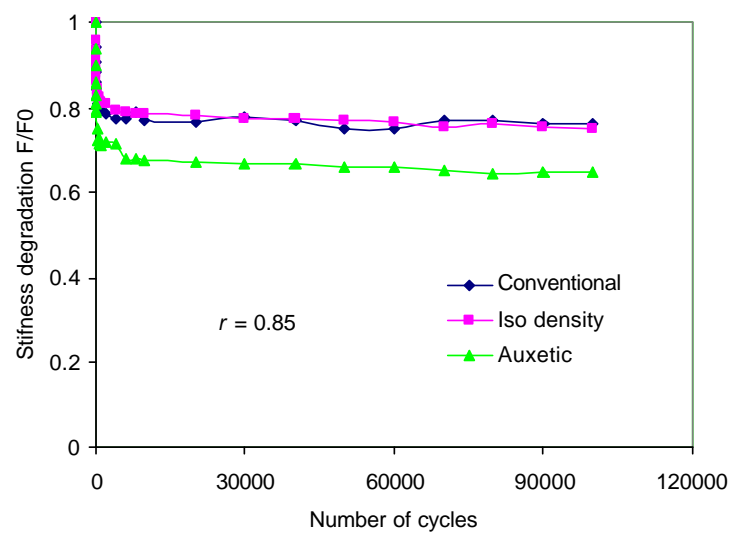

Figure 6. Stiffness degradation for $r=0.85$

One characteristic that is evident from the experimental results is the high damping capacity of the auxetic samples compared to the other foams for the same loading level. Fig. 7 shows the comparison of the energy loss per unit volume versus number of cycles for the three types of foams for a loading level $r=0.725$. While the conventional and iso-density samples similar values of energy loss per unit volume $\left(\sim 0.05 \mathrm{~mJ} / \mathrm{cm}^{3}\right)$ after 20000 cycles, the auxetic foam maintains an average value of $0.8 \mathrm{~mJ} / \mathrm{cm}^{3}$, with an increase by a factor 16 . However, for the highest loading level $(r=0.95$; Fig. 8), the iso-density foam start to differentiate considerably form the conventional one, with an average damping capacity after $10000 \mathrm{cycles}$ of $9 \mathrm{~mJ} / \mathrm{cm}^{3}$ compared to $2 \mathrm{~mJ} / \mathrm{cm}^{3}$ of the conventional one. The auxetic foam always provide enhanced damping capacity after the initial stage of stiffness degradation $\left(\sim 30 \mathrm{~mJ} / \mathrm{cm}^{3}\right)$, albeit with a factor of 3.3 compared to the iso-density one. The increase in damping performance of auxetic PU foams over conventional parent foam and iso-density one has been recorded in cyclic loading up to $10 \mathrm{~Hz}$ and $12.5 \%$ of offset with $5 \%$ of dynamic strain, where an equivalent viscoelastic complex modulus approach has been used to model the response of the cellular materials [11]. Increase in loss modulus for small harmonic peakto-peak deformations has also been observed in other compliant PU auxetic foams [21] and negative Poisson's ratio polymeric cellular solids obtained from Scott industrial parent foam[ 6]. The results presented in this work suggest that negative Poisson's ratio PU foams maintain a significant damping capacity over a large range of cyclic loading, both in terms of number of cycles and loading levels. 


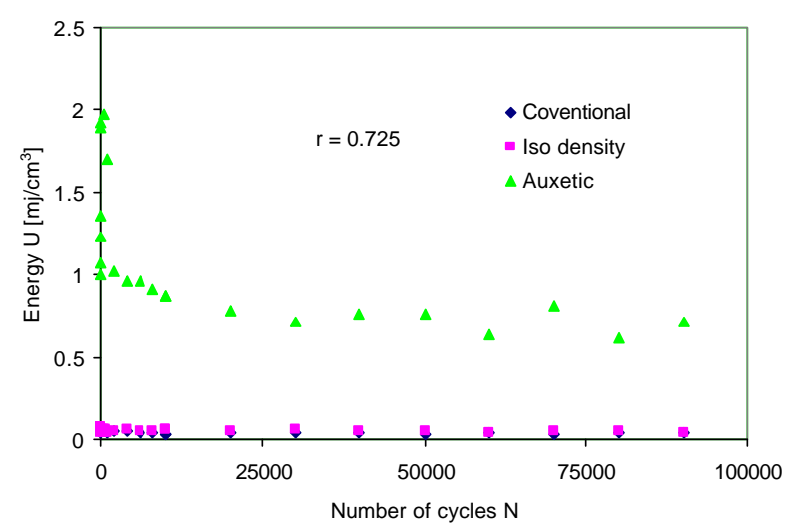

Figure 7. Damping capacity for $r=\mathbf{0 . 7 2 5}$

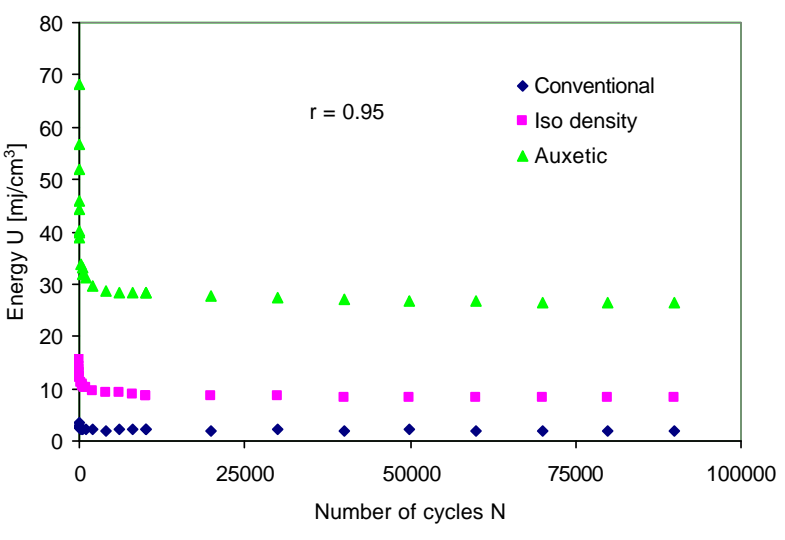

Figure 8. Damping capacity for $r=0.95$

\section{Conclusions}

In this study the dynamic characteristics of conventional open cell polyurethane (PU), of auxetic and of iso-density foams have been analyzed for possible antivibration glove applications and damping capacity usage. For the auxetic foams, the results suggested similar behavior to that previously reported in the research literature for auxetic foams, with significant dependence of the Poisson's ratio on the applied strain. The transmissibility tests, performed in accordance with the ISO 13753 procedure for resilient materials, suggested a strong dependence of the transmissibility on the foam manufacturing parameters. Within the frequency range from 10 to $31.5 \mathrm{~Hz}$ the transmissibility was found to be greater than 1, while it was less than 1 at all frequencies greater than $31.5 \mathrm{~Hz}$. Comparison of the transmissibility results to data available in the research literature for glove materials suggested that the foams tested in the current study provided values similar to the mean values for 80 resilient materials tested by Koton et. al., but were significantly higher than the five best materials (not all polymeric) identified by the same researchers. However, that the resilient behavior of glove isolation materials should also be evaluated in terms of the indentation characteristics. It can be hypothesized that a better distribution of the pressure at the interface between the glove and the human hand will result less damaging to skin tissue and to blood capillaries, even if associated with higher overall levels of vibration transmitted to the hand. In fact, according to Finite Element simulations on indentation resistance on auxetic open cell PU foams $[\mathbf{2 4}, \mathbf{2 5}]$, conventional foams present uneven distributions of the deformation fields. The auxetic foam, instead, tends to enfold around the indentation zone, providing a wrapping effect not present in the conventional cellular material. The normal stress distributions for the conventional and auxetic foam show a significant drop in terms of average normal stresses around the indentation area for the auxetic cellular solid, with an average decrease of a factor 3.2 present an average on the whole foam blocks.

A specific feature that the auxetic showed compared to the other foams was the significant damping capacity at various loading levels, with increase up to 16 times compared to the conventional foam. The iso-density one showed an enhanced damping behaviour versus the conventional parent foam only at loading level 0.93 , always scaled down by a factor of 3 compared to the auxetic one.

Further progress is on the way to design new types of auxetic foams with different phase parent characteristics, and benchmark them against available products in the market to assess both the energy absorption under cyclic loading and vibration suppression properties.

\section{Acknowledgements}

This work is supported by the grant EPSRC GR/R/97313.

\section{References}

1. Lakes R. S. Science 1987; 253: 1038-1040

2. Alderson KL, Simkins VR, Coenen VL, et al. Physica Status Solid B 2005, 242(3): 509-518.

3. Smith F. C., Scarpa F. and Chambers, B., 2000. IEEE Microwave and Guided Wave Letters 10(11), 451

4. Martz EO, Lakes RS, Goel VK, et al. Cellular Polymers 2005, 24(3), 127-138.

5. A.W. Lipsett, A. I. Beltzer, 1988, J. Acoust. Soc. Am., 84, 2179

6. C. P. Chen, R. S. Lakes, 1989. Cellular Polymers, 8, 343

7. B. Howell, P. Prendergast, L. Hansen, 1991. 'Acoustic behaviour of negative Poisson's ratio materials', DTRC-SME-91/01, David Taylor Research Centre, Annapolis, MD.

8. E. O. Martz, R. S. Lakes, J. B. Park, 1996. Cellular Polymers, 15(5), 349

9. C. P. Chen, R. S. Lakes, 1996. J. Engineering Materials and Technology, 118, 285

10. F. Scarpa, L. G. Ciffo, J. R. Yates, 2004. Smart Materials and Structures, 13, 49

11. Scarpa, F., Pastorino, P., Garelli, A., Patsias, S. and Ruzzene, M., 2005. Physica Status Solidi B, 242(3), 681

12. Cronjäger, L., Zeglam-Verch, I. And Gillmeister, F. 1992. Proceedings of the 6th International Conference on Hand-Arm Vibration, Bonn, Germany, 19-22 May, 747-754 
13. Smagowska, B. and Liwkowicz, J. 1992 Proceedings of the 6th International Conference on Hand-Arm Vibration, Bonn, Germany, 19-22 May, 755-764.

14. International Organisation for Standardisation 1999, ISO 13753:1999 Mechanical vibration and shock - Hand-arm vibration - Method for measuring the vibration transmissibility of resilient materials when loaded by the hand-arm system (Geneva: International Organisation for Standardization).

15. Rehkopf JD, Brodland GW, McNeice GM. Exp. Mech. 1996; 36(1): 1-6

16. Shen Y., Golnaraghi F. and Plumtree A. Int. J. Fatigue 2001; 23: 491-497.

17. Kanny K., Mahfuz H., Carlsson L. A. and Thomas T., Jeelani S. Comp. Struct. 2002; 58: 175-83

18. Kolsters H, Wennhage P. Proceedings of the Fifth International Conference on Sandwich Construction, Zurich, 5-7

September, 2000

19. Palissery V., Taylor M. and M. Browne. J. Mat. Sci: Mat. Med. 2004; 15: 61-67

20. Scarpa, F., Pastorino, P., Garelli, A., Patsias, S. and Ruzzene, M., 2005. Physica Status Solidi B, 242(3), 681

21. F. Scarpa, L. G. Ciffo, J. R. Yates, 2004. Smart Materials and Structures, 13, 49

22. SIGLAB 6.1 Users'Manual, 2001, Spectral Dynamics Inc., San Jose, CA

23. Koton, J., Kowalski, P. and Szopa, J. 1998. Proceedings of the 8th International Conference on Hand-Arm Vibration, 9-12 June, Umeå, Sweden, 329-335

24. Scarpa F, Giacomin J A, Zhang Y and Pastorino, P. 2005. Cellular Polymers 24(5), 253-268.

25. Wang Y. C. and Lakes, R., 2002. International Journal of Solids and Structures, 39(18), 4825 